\title{
Measurements in additive manufacturing
}

Pedersen, David Bue; Andersen, Sebastian Aagaard; Hansen, Hans Nørgaard

Published in:

Metrology

Link to article, DOI:

10.1007/978-981-10-4938-5_13

Publication date:

2019

Document Version

Peer reviewed version

Link back to DTU Orbit

Citation (APA):

Pedersen, D. B., Andersen, S. A., \& Hansen, H. N. (2019). Measurements in additive manufacturing. In W. Gao (Ed.), Metrology (pp. 369-397). Springer. Precision Manufacturing https://doi.org/10.1007/978-981-10-4938-5_13

\section{General rights}

Copyright and moral rights for the publications made accessible in the public portal are retained by the authors and/or other copyright owners and it is a condition of accessing publications that users recognise and abide by the legal requirements associated with these rights.

- Users may download and print one copy of any publication from the public portal for the purpose of private study or research.

- You may not further distribute the material or use it for any profit-making activity or commercial gain

- You may freely distribute the URL identifying the publication in the public portal

If you believe that this document breaches copyright please contact us providing details, and we will remove access to the work immediately and investigate your claim 


\section{Measurements in additive manufacturing \\ Pedersen, David Bue; Andersen, Sebastian Aagaard, Hansen, Hans Nørgaard;}

\section{Introduction}

Additive manufacturing ( $\mathrm{AM}$ ) is a palette of technologies that colloquially is denominated by the common appellation of 3D Printing. AM has created a strong linkage between the digital and physical manufacturing, thus nourishing by its nature a wider trend; the digitization and the automation of the manufacturing industry. For this reason, the increasing adoption of AM within the manufacturing industry is pushing companies to research new ways of adapting their manufacturing models and optimize their manufacturing strategies by integrating these manufacturing technologies of tomorrow into existing production and bolster their strategies towards a digital to physical conversion.

This trend sets new demands with respect to how measurements are carried out when these relate to components manufactured by AM. Such measurements can be of dimensional as well as of physical classification. It is rare that $A M$ can stand alone as a manufacturing method. As a minimum validation during pre- and post manufacturing tasks must be handled conjointly with the additive manufacturing task. These tasks are both digital and physical nature and include, job preparation, machine preparation as well as post processing by cleansing and part treatment for the component to acquire its final properties and dimensions. These activities and their protocols, will directly affect- and in many cases dictate the final part quality and dimensions and must be understood and taken into account when setting up manufacturing based upon any of the industrialised AM methods. In this chapter all of the aforementioned prerequisites for a controlled additive manufacturing process are addressed and considerations are given for ensuring a reliable manufacturing.

By and large, standardisation of procedures for suitable approaches to measurements within Additive Manufacturing is an on-going task. This means that for many validation procedures no designated standard method exist to aid the validating body in adhering to correct protocol. The works carried out in standardisation for Additive Manufacturing is predominantly carried out within Committee F42 on Additive Manufacturing Technologies of the ASTM body as well as the corresponding ISO/TC 261 body. ISO/TC 261 and ASTM F42 have formulated a Joint Plan for Additive Manufacturing Standards Development wherein four AM standard tracks are being pursued as listed below. Non-designated ASTM/ISO standards are working titles for the Standards Development efforts currently taking place.

\section{Terminology}

- $\quad$ ASTM F2792-12a

Standard Terminology for Additive Manufacturing Technologies, (Withdrawn 2015)

- ISO/ASTM 52900:2015 (ASTM F2792)

Additive manufacturing - General principles - Terminology

- ISO 17296-1

Additive manufacturing - General principles part 1: Terminology

- ISO/ASTM 52921-13

Standard terminology for additive manufacturing - Coordinate systems and test methodologies

\section{Process / Materials}

- ISO 17296-2

Additive manufacturing - General principles part 2: Overview of process categories and feedstock

- Qualification and Certification Methods

- Requirements for Purchased AM Parts 
- Non-Destructive Evaluation Methods

Test Methods

- ISO 17296-3

Additive manufacturing - General principles part 3: Main characteristics and corresponding test methods

- Test Artifacts

- General Test Methods

- Performance Test Methods

Design / Data Formats

- $\quad$ ISO 17296-4

Additive manufacturing - General principles part 4: Overview of data processing

- ISO / ASTM 52915-13

Standard practices - Guidelines for design for additive manufacturing

- Data Structures and Metrics for AM Models

\section{Physical and digital elements of additive manufacturing}

By virtue of the highly digital nature of industrialized AM processes, the digital aspect must be taken into account when measuring and planning measurement strategies are taken to protocol in a manufacturing environment. Figure 1 serves as an overview of these elements for a generic AM process. All potential error sources related to the physical and digital process chain are listed in white slanted boxes.

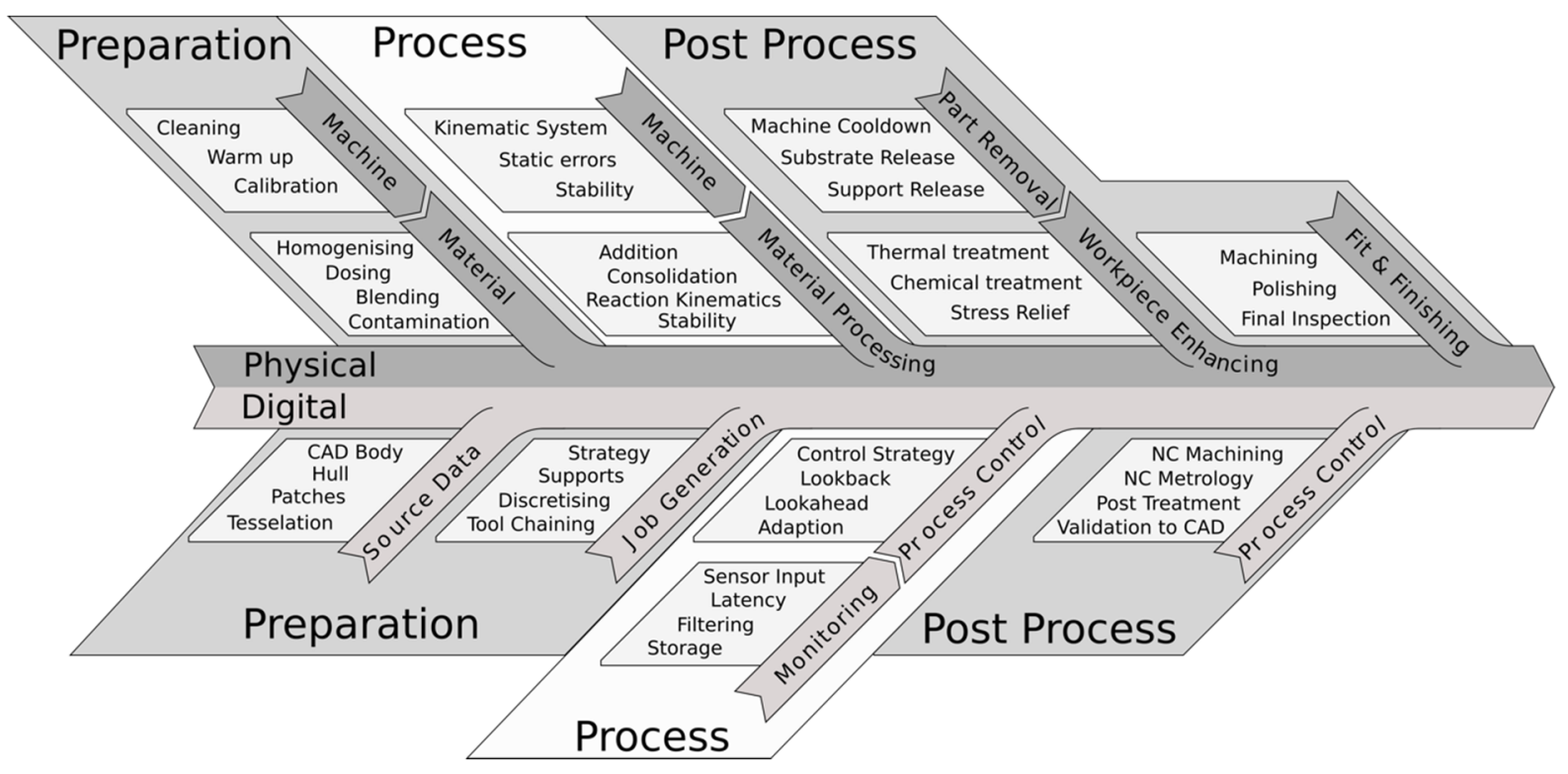

Figure 1, overview of physical and digital links of an AM process chain

Prior to manufacturing, the quality of the source 3D geometry and the digital manufacturing planning through job generation is equally decisive to the manufactured component quality, as physical tasks involving machine set-up and materials preparation. This in part given that the de-facto standard describing geometry for AM job generation is a tessellated format (.STL) by which a CAD geometry is approximated by a faceted wireframe with limited fidelity. During processing, the in-process, monitoring and control capabilities of the AM machine-tool is equally decisive to part quality as the physical machine design and material processing capabilities. This, as most industrial AM processes, 
needs to be highly controlled by active control loops in order to sustain stable process conditions. During post-processing the component acquires its final geometry and physical properties. These processing steps can therefore be argued to be the most critical to oversee and regulated by e.g. NC driven technologies such as NC Machining and NC metrology. Thus, the entire AM workflow is a physical materials interaction where strategies, in-process decisions and post treatment is tied by a digital information layer that must be tightly overseen to produce measurable and functional components.

\section{Precision Additive manufacturing}

The Additive Manufacturing family covers technologies that are either predominantly employed for industry-grade manufacturing or as technologies that are pertinent to prototyping application. Requirements to functionality and precision are usually less for the latter group, as deviations often can be better accepted on prototype grade components than for production grade components. By virtue of this discretising, Precision Additive Manufacturing in the context of this chapter pertain to those technologies that primarily are applied for industrial manufacturing purposes. These technologies are those which rely on photo initiated polymerisation of plastic- and polymer resins as well as those by which metal is consolidated to form near-net-shape components. The latter where the outcome of the additive process can be regarded as workpieces for a final machining and/or polishing operation to conform with the net-shape geometry and functional surface specification of the part.

\subsection{Photo initiated polymerisation}

Photo initiated polymerisation is a mechanism that is also known as photo polymerisation in which a light induced crosslinking mechanism is harnessed in order to cure a polymer resin. In what follows, the mechanism will be denominated simply as ' UV curing' given most industry grade materials for AM is photo initiated by irradiation within the UV spectrum. UV curing is a process where a polymer resin (i.e. multifunctional monomer and oligomer matrix) is transformed into a crosslinked polymer by a chain reaction initiated by reactive species known as photoinitiators that upon irradiation form free radicals or ions as shown in figure 2 . The role of the photoinitiator is that most monomers or oligomers do not produce initiating species with high yield to react with UV light. Thus, the photoinitiator needs to be added to the formulation. Depending on the photoinitiator, the photopolymer can be engineered to react to other ranges of wavelengths (visible light) if this is a wanted property $[1,2]$. Most industry-grade photopolymers are engineered to be reactive in the UV spectrum since the shorter wavelength of coherent UV light (with respect to visible light) allows for smaller features to be generated as a function of the favourable beam shaping properties of a monochromatic UV light source.

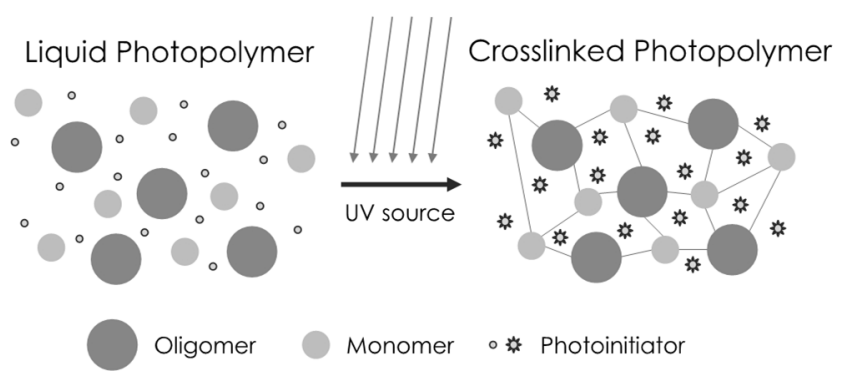

Figure 2, the principle of photo polymerisation

\subsection{Photo polymer based AM methods}


Additive Manufacturing methods that rely on the selective photo initiated polymerisation of polymer resins held in a resin container are known by numerous proprietary names yet all fall under the denominator vat photo polymerisation as given by the ISO/ASTM 52900:2015 naming convention. These processes rely on either the digital driven scan-line rasterising of an image by means of a laser point source or on the projection of image masks from a projection source to selectively consolidate the photopolymer to the workpiece. Two primary machine configurations exist; a top-down and a bottom-up configuration as seen in figure 3 .

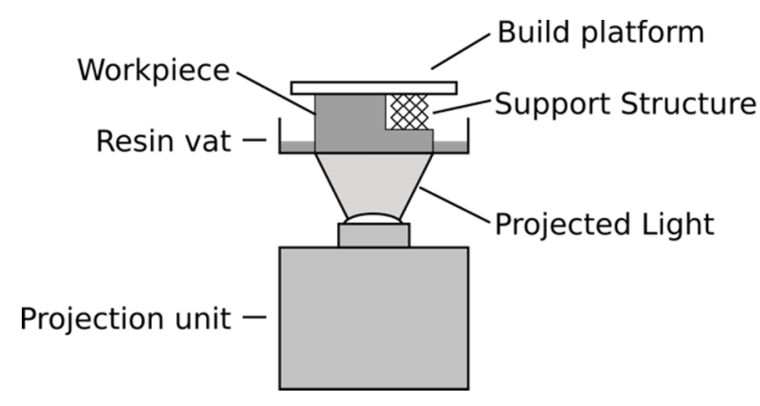

(A) Bottom-up

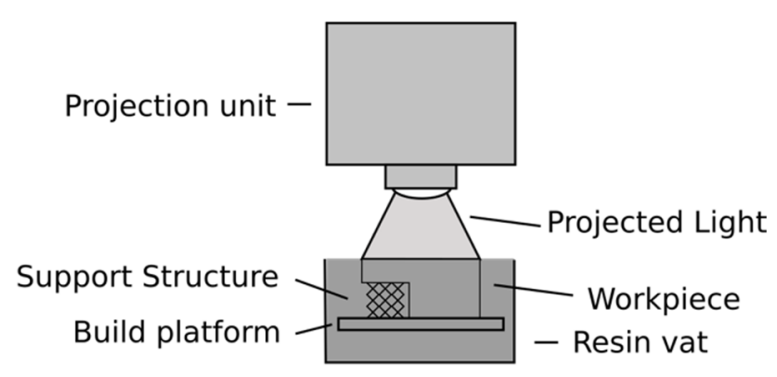

(B) Top-down

Figure 3, (A) Bottom-up configuration; (B) Top-down configuration of a vat photo polymerisation machine tool for additive manufacturing.

\subsection{Metal Powder Consolidation}

Metal Powder Consolidation is the underlaying working principle for a family of processes by which metal powder is selectively consolidated to the near-net shape of a component by either sintering or melting. The consolidation is predominantly induced by means of a high powered laser and in an inert atmosphere yet some metal powder consolidation machine tools operate by means of a an electron beam in a high vacuum atmosphere. It is noticable that unlike the previously described photopolymerisation methods, not only geometry but to a high degree also physical properties of the component can be structured in-process. These include density, hardness microstructural composition, stress distribution and similar properties that can be altered through thermal treatment of metal. Thus the outcome of any given metal AM process chain is the most complex ramification of the elements presented in figure 1. Both geometry and material is given their substance by the swift fusing metal particles, and rapidly progression of the heat source away from the treated area introducing quench-like cooling. As successive layers are built atop, reheating of the already consolidated matter occur. The outcome of this discordant by-layer process are components that in most instances narrowly meet near-net shape, and a similar system as that of tolerance grades and machining allowance grades known from cast metals and their alloys [3] must be used to accommodate allowance for a subsequent machining procedure. Concequently final quality assurance of the component follows the methods that are defined for machined parts and surfaces [4].

\subsection{Powder Consolidation methods}

Production of AM metal parts via powder consolidation can follow two strategies. First is sintering, which requires the material to reach temperatures near melting temperature at which the powder fuse to a porous matter with a microstructure that is inherited from the packing of the powder prior to consolidation. Second is a full melting of the powder by which the powder is heated to temperatures above the melting point of the powder. Hereby a melt pool is formed and the resolidification of the melt expunge the traces of the porous powdered initial state of the process material, thus forming dense solid matter. Sintering is generally known to be a cleaner process with 
less formation of spatter and better surface fidelity yet traded off by more porous and weaker material structure.

\subsubsection{Sintering}

Sintering bonds particles at their interface and thus forms a solid (porous) structure, through heating without melting (alternatively in combination with, or by pressure). Solid-state sintering occurs when a powdered material is heated, and is consequently the mechanism applied in sintering based metal AM. Adjacent powder particles will start merging, and bond through diffusion, as shown in figure 4 . After bonding, the bonded bulk material will experience a change in global properties, such as strength, density, ductility, and thermal/electrical conductivity. The nature of the sintering process makes it prone to the formation of pores medially between the surrounding particles.

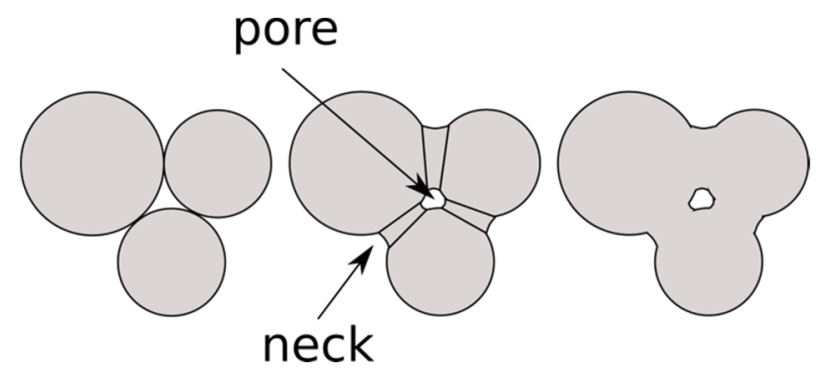

Figure 4, the principle of solid state sintering

This too is illustrated in figure 4. Here the particles shown merge during the sintering process, and neck growth occurs between the former separate metal particles. Finally, shrinkage occur, by which, during the neck growth the center parts of the particles move closer together, and the compaction of the particles into a solid makes the total volume shrink as compared to the original state [5].

\subsubsection{Melting}

As opposed to sintering, the consolidation of the powder particles by melting is done in a way that ensures that the targeted particles are fully molten, as seen in figure 5 . This approach is more difficult to control since minute changes in heat flux from the heat source affect the size of the melt pool, affecting overhanging structures and in general the down-face of the manufactured component. Whereas sintering within AM is not exclusive to metals, characteristics of metal alloys such as conductivity, and a distinct melting point, makes them de-facto exclusively used as materials for full melting in powder consolidation systems. The rapid melting and solidification enabled by the process, allows for parts with tailored properties, distinct from those obtained from traditionally processed parts [6]. This facilitates the exploitation of localized material properties, obtained from refined microstructures. Formation of non-equilibrium phases, and supersaturated solutions. Second-phase particles such as inclusions and carbides, or extremely fine, refined microstructures, are side effects of the process.

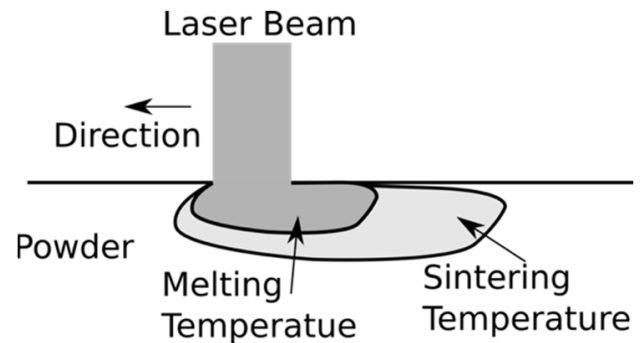

Figure 5, The principle of full melting of a workpiece 
Due to the similarity of powder based sintering and melting, whether the technology is referred to as sintering or melting, often both of the states are present. Given that the sintering temperature is slightly below the temperature at which a melt pool is formed, as shown in figure 5 , sintering will occur in the boundary of a geometry made from a melt driven process. Similar to this, localised melting can occur during a sintering process, where impurities and process fluctuation may temporarily lead to the formation of melt. Melting is often preferred over sintering given that, in order for the process to be as economically competitive as possible, the fusion of the powder should happen as quickly as possible in order to increase the build rate. This is achieved by a fast-moving energy source, with a high-energy flux, and fusion by melting. As fusion by sintering alone, typically takes much longer time. Hence, the reason for most AM processes, not to use sintering as the primary mechanism of fusion. Nonetheless, sintering still plays secondary roles in the process [7].

\subsection{Powder Consolidation based AM methods}

Two primary machine tool types exist for the additive manufacture from metal powders. They differ by design by one process employing a powder bed in which powder is consolidated by either sintering or melting and where the process can occur either in a vacuum or in an inert atmosphere. The other method employs a cladding technique where a freely movable laser nozzle delivers a powder spray in a carrier gas to the workpiece.

\subsubsection{Cladding}

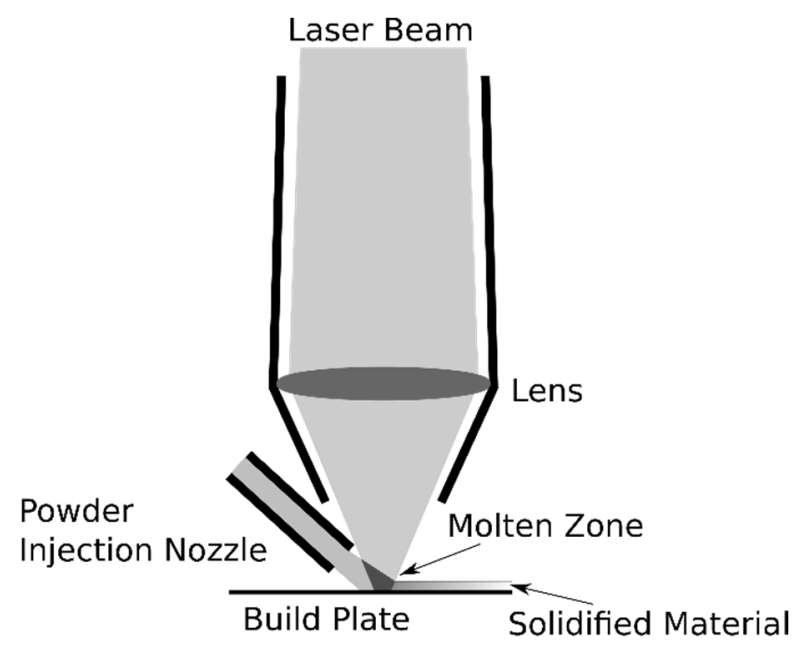

Figure 6, The principle of laser cladding

The cladding principle is illustrated in figure 6. A tooling system comprised of an integrated two-stage nozzle system function to deliver a steady stream of fluidised powder to the workpiece while also delivering the heat flux in the form of focused laser light to the workpiece. Fluidising the powder and bringing it into the beam of the laser is done by means of an inert carrier gas spraying the powder directly to where material is required. The laser beam intercepts the powder stream at the desired deposition location, and fuses the melt directly onto the work piece. The nozzle is mounted in an NC machine tool and containing both sprayer and typically an optical delivery fibre fixing their location relative to each other, ensuring the correct focal distance of the laser. Most systems employ a 5-axis NC machine tool that allow for rotation of the workpiece such that the cladded material is deposited with the force of gravity hereby minimising the need for support structures. Support structure serves primarily to fix the workpiece such that thermal residual stresses does not allow for bending of the workpiece to occur. Many cladding systems are integrated on 5 axis milling machine tools, allowing for hybrid manufacture such that internal surfaces can be machined before these are closed off and inaccessible for post machining. Given the free-form nature of cladding, layers that are cladded to the 
workpiece does not need to be planar and the process is ideal for adding material to an existing workpiece that has been made from conventional manufacturing for either finishing the workpiece or for repair purposes.

\subsubsection{Powder Bed Fusion}

The most common machine tool for metal additive manufacturing is the powder bed fusion systems. This type of machine operates by selectively fusing powder following a by-layer deposition principle. Powder is contained in a silo and fed to a re-coater that coat the build chamber of the machine with an evenly distributed layer of powder between consolidation operations. The powder delivery system is responsible for relocating the build plate along the z-stage, and therefore defines the accuracy and resolution of the build in the vertical direction. The tool distributing the powder evenly can be in the form of a scraper, a roller or a blade that is designed to both fluidize and distribute the powder. As this tool has major influence of the distributing of the layer the powder and the tool has to be carefully matched to the powder and the application. When the layer is in place, the laser beam is selectively manoeuvred to consolidate the metal powder particles in order to create the desired geometry as seen in figure 7.

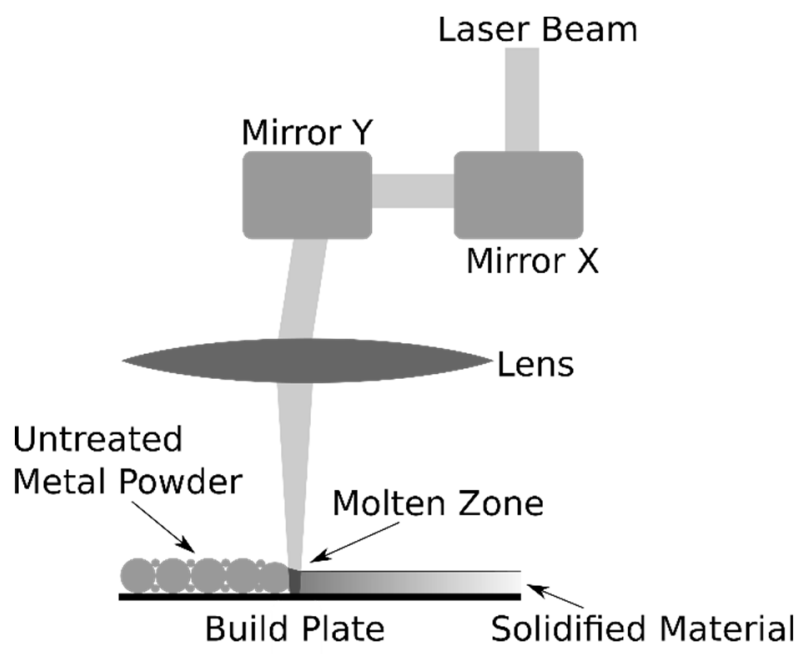

Figure 7, The principle of powder bed fusion

As the only moveable part in the consolidation process is the laser beam, the control is done by a scanner mirror system. Typically, two separate mirrors mounted onto galvanometric motors are used to rotate each mirror. The mirrors are then located in a way that allows discrete movement along the $x y$-plane. This is a consolidation process where support structure is added solely to counter thermal residual stresses, as the untreated powder will stay in the build chamber and support any overhangs presented.

\section{Measurements in Additive Manufacturing}

Discrepancies of photo-polymerisation and powder consolidation lend to contrasting focus for validation of parts as manufactured, and in advance of post processing. The high fidelity and material homogeneity of photo polymer components allow for net shape manufacturing and measurements can be taken similar to measurements in the thermoplastics processing industry. These measurements are typically those of shape, geometry and surface and serve foremost to quality assure the manufactured geometrical tolerances. The methods here fore can be tactile or optical, can range over simple control measurements using hand measurement devices such as callipers and micrometre dial 
gauges, to complex validation using optical CMMs or 3D scanners. The latter can further contribute to measurement effectiveness when dealing with a high geometrical complexity such as e.g. the manufacture of hearing aids which is one of the largest industrial use-cases for photo-polymer components.

Metal components made from consolidation of powder are rarely manufactured directly to tolerances and net shape. Given the poor fidelity of components as manufactured in the additive machine tool, the components are produced with machining allowances for subsequent fit and finishing. In many use-cases the components are blatantly regarded as feedstock for a complex thermal and mechanical process chain that finishes with NC machining, grinding and polishing of the component to reach net shape. Measurements following the additive manufacture serve therefore primarily to ensure that the material and inaccessible internal structures are manufactured as expected. Thus, the tool of choice is often, and in some cases solely, to be using CT scanning for the validation procedure. This, however, is not the only suitable approach to measurements for powder based metal additive manufacturing and alternative validation methods will be presented in the following. It is ultimately worth to bespeak that measurement of complex internal and inaccessible areas of any additively manufactured component can only be non-destructively measured by penetrating scanning methods such as CT scanning. Albeit the discrepancies of photo polymerisation and powder consolidation manufacturing methods, a general classification for measurements within additive can be segmented as follows, and will be used in section 5 and 6 :

- Machine errors - Measurements relating to the validation of the additive machine tool e.g. spindle errors, axes errors including translational, rotational and squareness errors,

- Shape / Geometry - of the manufactured component,

- Surfaces- of the manufactured component,

- Material (properties) - of the manufactured component.

\section{Measurements in additive manufacturing - photo polymerization}

This section covers an array of common errors that is related specifically to components made by vat photo polymerisation. Some of these errors are inherent to the machine tool whereas others are inherent to the process of selective cross-linking of the photopolymer. These errors must be understood before appropriate validation methods can be selected. The errors can be divided into:

- Machine Errors

- Geometry, Shape and Shape Deformations

- $\quad$ Post Treatment Errors

Typically material properties in these types of processes are related to post treatment of the parts.

\subsection{Machine Errors}

All common photopolymer based systems are electro-opto-mechanical systems, thus errors are originating from these three main system components.

\subsubsection{Electro}

For scanning laser systems, the galvanometer scanning system is discretised to an extent where motion errors are predominantly caused by overshoot, ringing and linearity similar to errors found in powder bed fusion systems as described in section 6.1.1. For mask projection systems, an error component is introduced to the horizontal plane of the build envelope of the machine tool. This error is inherited to the 
discretization from geometry to the limited pixel resolution available on the Digital Mirror Device (DMD) micro mirror chip array that is used for image generation as shown on figure 8.

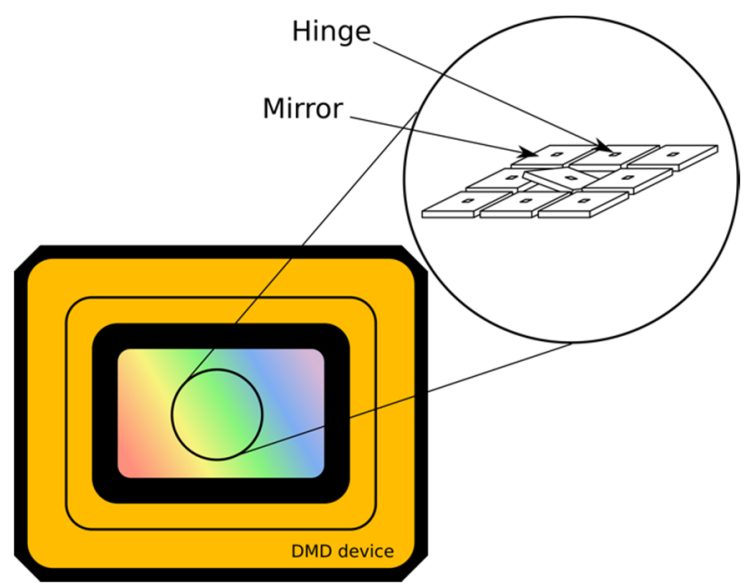

Figure 8, Design of a DMD micro-mirror array

Furthermore, local damage to one or more mirrors of the DMD device can produce a "dead" pixel which will be either always on or always off due to damage to the mirror pivoting mechanism.

Since all geometry is a best-fit within the pixel discretisation of the DMD mirror device, geometry e.g. a circular shape will be resolved into a corresponding pixel matrix as seen on figure 9 below. Already at this stage a roundness error is introduced. In this example, a circular feature covering 14 (left) and 15 (right) pixels are resolved to the pixel matrix on figure 9. The introduced roundness error is 0.856 and 0.902 for the 14 and 15 pixel diameters circles respectively.

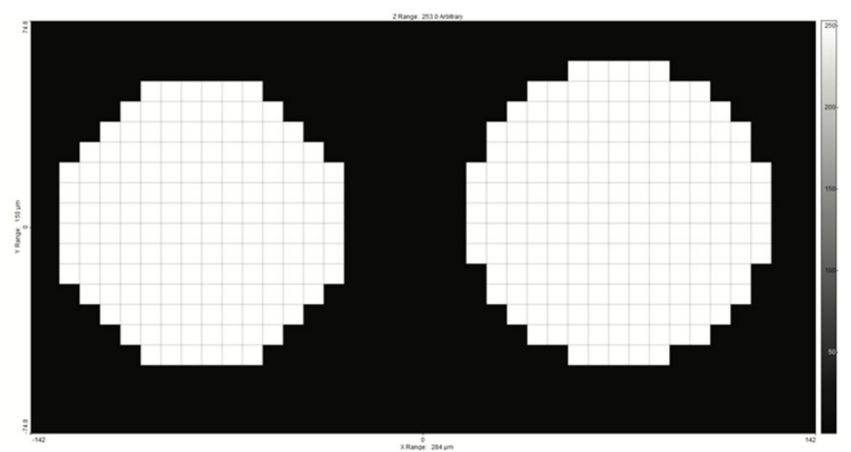

Figure 9, Pixilation introduced by resolving a circle of 14 (left) and 15 (right) pixels onto a DMD device

\subsubsection{Mechanical}

The vertical stage of any photopolymer system is subject to the same errors as are known from the characterisation of common motion stages, with the same methodology to describing the accuracy and repeatability of the stage. These error components are reversal error, slack, runout (straightness), angular runout (pitch/yaw/roll), eccentricity, wobble, drift, load capacity (transverse, normal, axial), inertia, jerk and the alignment of the stage to the image plane of the system.

\subsubsection{Optical}

Optical errors are errors introduced from the optical path from the light source to the 
image plane of the system. These errors manifest themselves as distortions whish are the deviation of the image from the ideal rectilinear projection. The distortion field is comprised by two main components; tangential (skewness) caused by misalignment of optical elements to the optical axis of the lens system and radial (barrel or pincushion) distortions, though the radial distortion field is minimal in modern lens systems (caused by lens geometry approximation). Furthermore, optical aberration from imperfections in the grinding and polishing of the lens elements of the projection system may induce localised errors and errors such as local defocusing. [8]

\subsection{Shape and Shape Deformations}

Shape errors will occur during manufacturing. These relate predominantly to two main constituents; from shrinkage during solidification and from plastic deformation during layer change.

Whereas thermoplastic materials have a branched or linear molecular structure, which allow them to be melted and solidified repeatedly. For cross-linked polymers, they do not melt exhibiting less creep and stress relaxation as a result. There are two types of photopolymer chemistry: free-radical (acrylates) and cationic (epoxy and vinylether).

The early resins created for vat photo polymerisation was solely comprised from acrylate based resins, which has high reactivity (fast reaction when exposed to UV light) as well as a high shrinkage rate (5 20\%) [9]. Due to the fast solidification and resultant little relaxation time stresses build up rapid in the photopolymer than for the epoxy based resins that cure slower allowing for relaxation during solidification [10]. Therefore, acrylate resins are prone to poor geometrical and mechanical performance, not solely from the high shrinkage, but also from lack of relaxation during processing as a result of the fast curing. Epoxy based resins on the other hand, have low shrinkage (1-2\%), lower solidification rate and thus less tendency to curl and warp. Current photo polymers are complex blends of methacrylates, epoxies and polyesters designed to yield good overall performance with respect to procesability and performance in their cross-linked processed state. These blends shrink typically $4 \%$ [11]. Irrespective, given the layered nature to vat photo polymerisation, as each layer contracts upon manufacture the resultant shrinkage leads to stress build-up in the component that will result in component warp. This is seen most clearly on wide components that are manufactured laying down parallel to the build plane and on sides facing the build platform. A critical example of warping geometry can be seen in figure 10 .
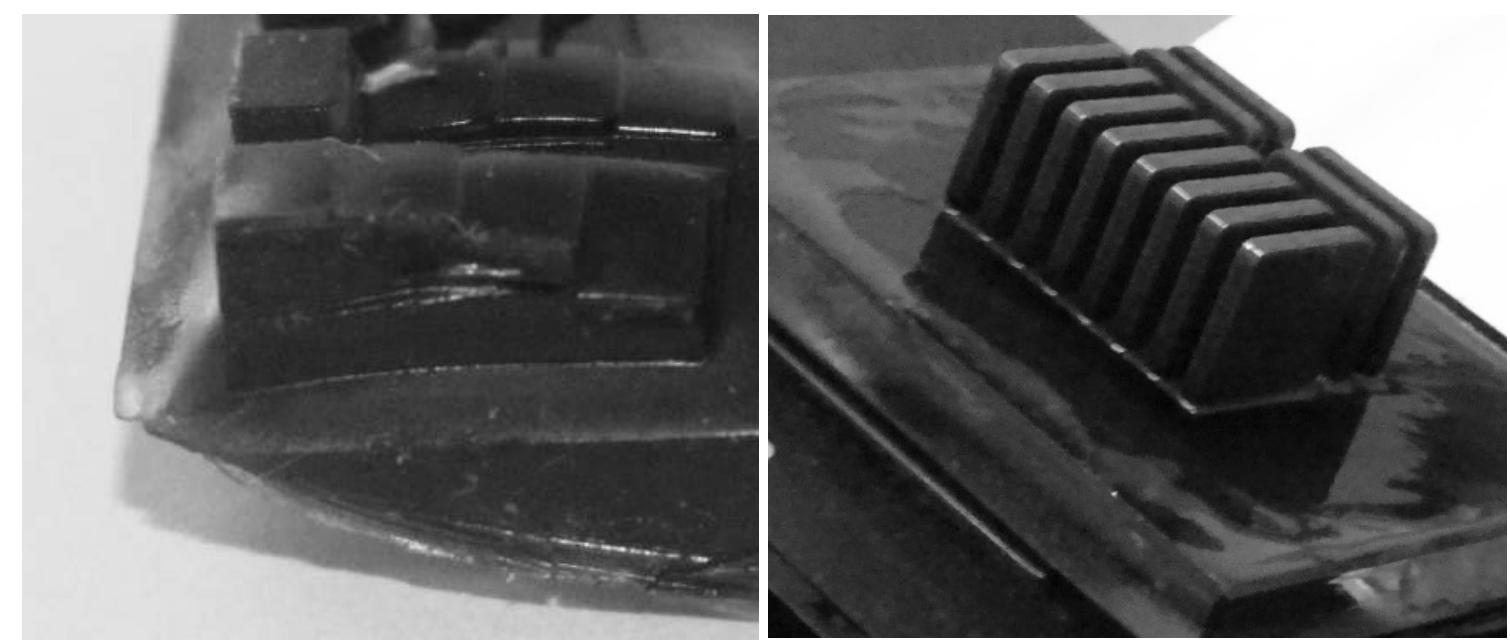

Figure 10, left, an example of high warp of an acrylate photopolymer. right, a modern photopolymer blend with low shrinkage and orthogonal part orientation to minimize warp. 
Errors relating to plastic deformation during layer change is induced from the solidification strategy that make layered photo polymerisation possible. Solidification of photopolymer onto a fully cured previous layer result in an interface zone where adhesion onto the previous layer is weak. This result in poor mechanical properties with high risk of delamination occurring. Therefore, any layer during the fabrication is only irradiated with an image mask for the duration it takes for the cross-linking to form a stable layer. This can be regarded as a layer of hard gel. Upon layer change, for bottom-up machine tools this layer will be under tension as the gelated layer is being lifted off the exposure window in the bottom of the vat, leading to plastic deformation. Upon repositioning the layer will be under compression as the photopolymer film between component and exposure window is being squeezed away. This type of effect can be seen in figure 11 .

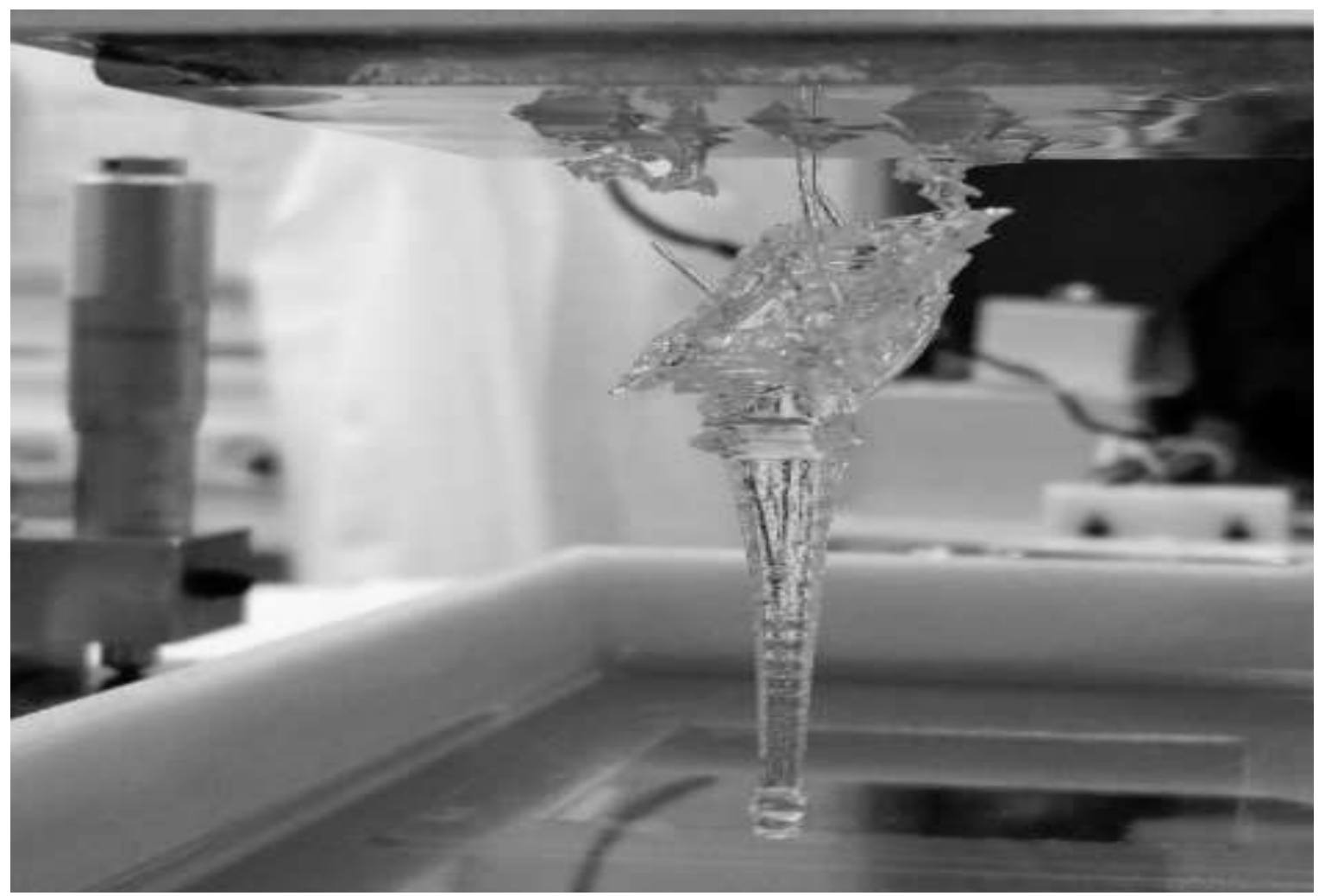

Figure 11, Severe forces during layer change on a bottom-up style vat photo polymerisation platform causing plastic deformation leading to critical manufacturing failure

For top down machine tools, the gellated layer may be affected by the recoating action of the wiper. For top-down machines the effect is less prone to cause geometrical deviations on the part.

\subsection{Post Treatment Errors}

Post-manufacturing treatment of photopolymer components is critical for the manufacture of components that conform with tolerances. This both relates to dimensional and physical tolerances. Post-manufacturing treatment include the following procedures where tolerance deviations can be induced:

- Washing and Cleaning

- Support Removal \& Post Curing

- $\quad$ Fit \& Finishing

\subsubsection{Washing and Cleaning}

Washing and cleaning serve to remove the thin film of unsolidified photo polymer that will cling to the 
manufactured geometry. This process involves the use of a solvent to dissolve the film as well as a washing that can be either through agitation via ultrasound and/or rinsing through circulation of the solvent. A commonly used solvent is Isopropanol which has good solubility compatibility with most industrially employed photopolymers. Washing and cleaning may lead to a whitening discolouration of the component from the chemical interaction with the solvent that can be prevented by following the washing procedure with a treatment of the component by briefly submerging it into propylene glycol. The treatment of the component with these chemical compounds will result in that the component will swell. A temporary effect that require the component to rest until swelling has diminished before further processing. It is worthwhile to notice that alcohols are prone to cause stress crazing of acrylic materials. Given most photo polymer blends contain acrylic compounds, surface cracking from post treatment can be observed and typically relates hereto [12]. Figure 12 shows how surface crack occurrence can be seen in poorly washed and cleansed photopolymer components.

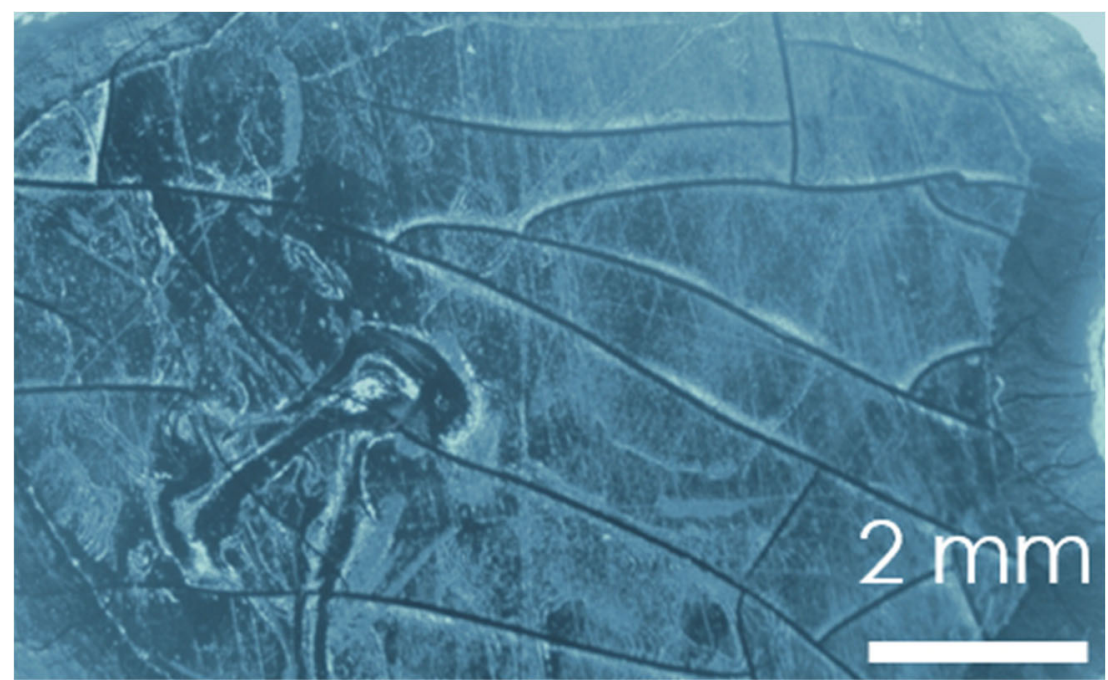

Figure 12, Surface cracks during post-treatment due to alcohol induced stress crazing and/or aggressive light induced post curing

\subsubsection{Support Removal \& Post Curing}

Post curing is the process by which the washed and cleaned component is given its final mechanical properties, where the component is cured to an extent where it is chemically safe for direct skin exposure and where the curing induced change in stress concentration within the part may result in that the component deform excessively. One of the most often seen errors induced by this process is that not all residual photopolymer from the cleaning procedure has been removed from e.g. high aspect ratio holes, crevices and internal tunnels. This matter will be solidified by post curing and will render these features incomplete spite correctly produced during manufacturing. Assuming correct cleansing, errors can be minimised during post curing by leaving support on the component to reinforce it during the change of stress concentration as a result of the post curing. A slower post curing by flood light or by heat induced post curing is to favour over aggressive flash light curing since the former methods will allow for the components to undergo relaxation during the treatment. Support removal should be carried out by cutting and polishing over breaking off, to limit scarring on surfaces in contact with the support structure.

\subsubsection{Fit and Finishing}

Many industrial photo polymer components e.g. hearing aid shells undergo a final automated fit and finishing procedure that involve automated polishing. This is most often done by a rotary tumble polishing machine. If such procedures are used for the component to be validated, rotary tumble polishing and its effect is well studied in literature and should be taken into account. 


\subsection{Surfaces in photopolymer components}

A well performing vat photo polymerisation platform is capable of producing high fidelity components and allow for embedding of complex three dimensional micro features and the manufacture of multiscale components. Such a component, manufactured to recreate a bioinspired hydrophobic surface can be seen on figure 13. On such components, it can be difficult to appoint when to distinguish amongst form and surface [13]. Thus, simple surface measurements such as profilometry are unsuited to describe the component and advanced methods such as confocal microscopy $\mu$ SEM and 3D LDOF microscopy.

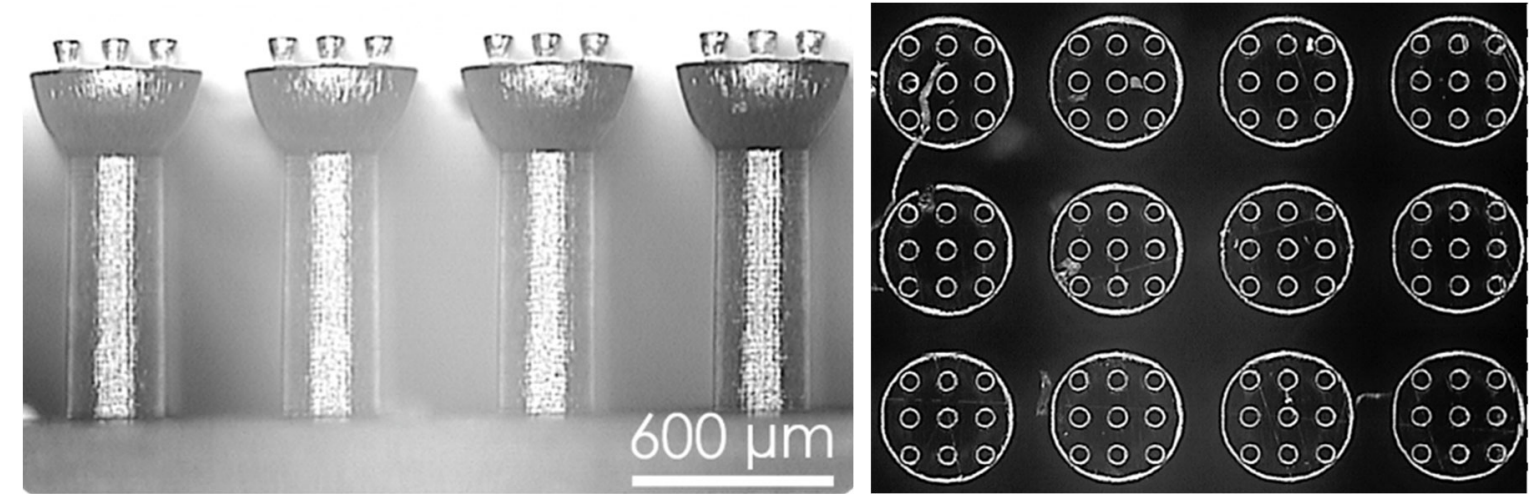

Figure 13, optical microscopy of a component with a multi-scale micro structured surface made by vat photo polymerisation [13]

Advanced methods, spite their capabilities to map surfaces with high fidelity, can prove inadequate, as can be demonstrated with the exhibited multi-scale micro structured component. Figure 14 shows an attempt at mapping the component with an 3D Focus-Variation microscope. Inspecting the component at an acute angle results in numerous blind-spots of the stem of the structure which force upon conducting the inspection as a normal to the surface. Hereby side walls are obstructed from view. Thus, multiple measurements from complementary angles must be carried out in order to fully map the surface.

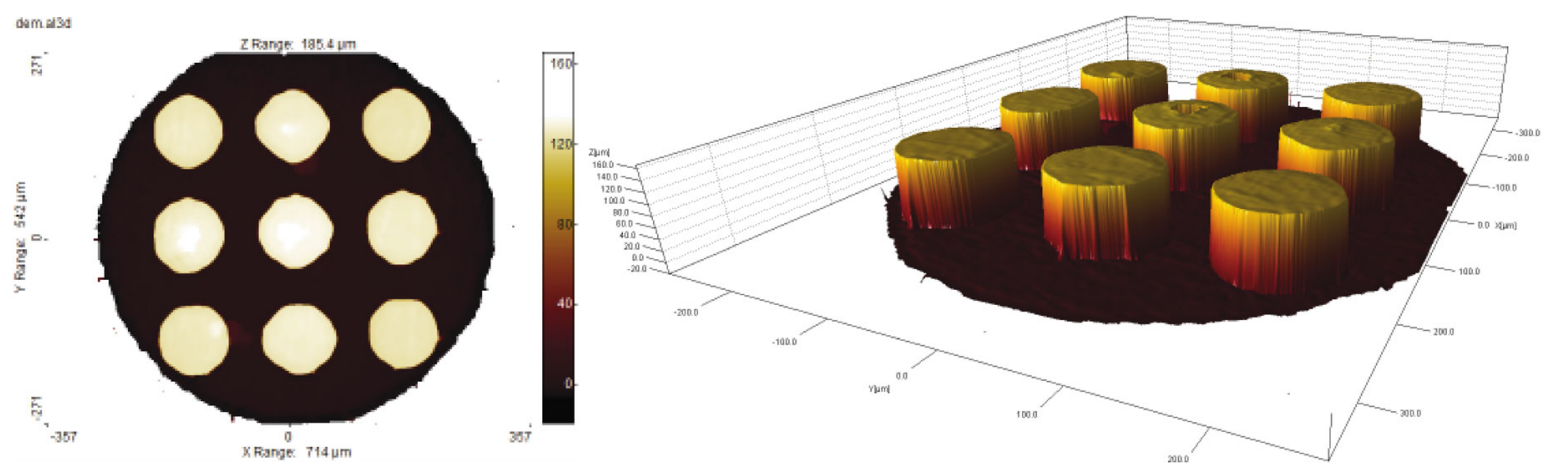

Figure 14, Focus-Variation microscopy of micro structured surface manufactured using photo polymerization [13]

Certain microstructural features are inherited from the photo polymerization process itself. In section 5.1.1, it was described how arcs are discretized at image mask level. If a thorough focus calibration is carried out on the DMD enabled vat photo polymerization machine tool, this pixelated approximation is visible on microstructure level on the manufactured component as in figure 15. 

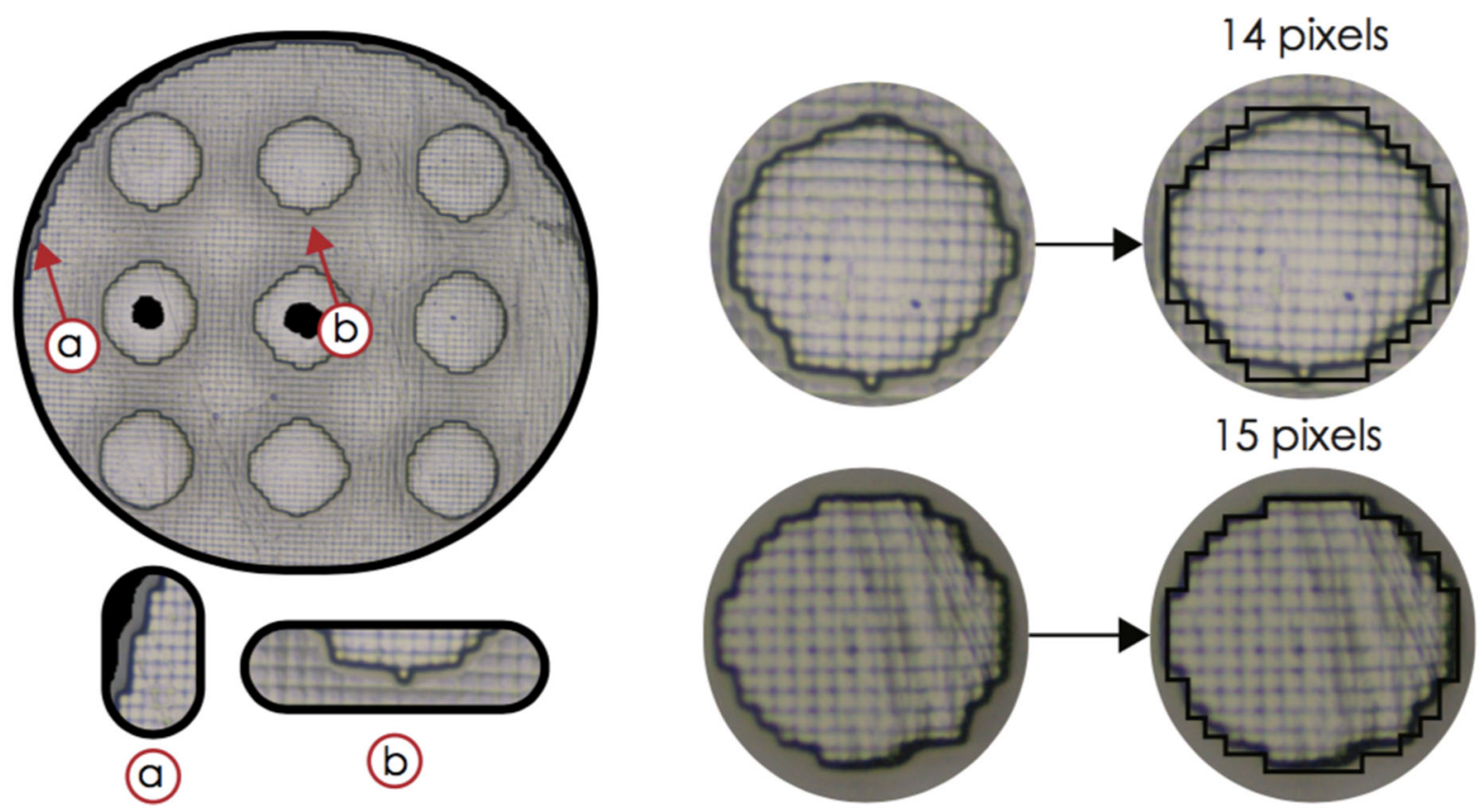

Figure 15, Cylindrical $150 \mu$ m features on a sample where discretized at image mask level to the cylindrical feature is visible. [13]

This can be regarded as an inevitable surface characteristics that is similar to e.g. the periodic surface profile of a component turned on a lathe. As with a turned component where the periodic surface can be used to find the transversal feed of the carriage, the pitch of the pixel grid can yield valuable information of the state of optical system on the vat photopolymerisation machine tool. Below, a surface microscopy image was processed by identifying the pixel boundaries with their grey-scale information, it was possible to measure each pixel as shown in figure 16 . The machine tool has a nominal pixel size of $7.6 \mu \mathrm{m}$. Edges of the tip had an average of $5.96 \mu \mathrm{m}$ of pixel replication on the surface which is smaller than the nominal. This effect is from that the very edge of the inspected feature is not fully cured, due to a combination of light scattering and light intensity drop on the edges of each pixel. The pixels found in the centre of the inspected feature have an average of $7.34 \mu \mathrm{m}$, slightly smaller than the nominal value. This stems in this specific case from the optical system since the optical plane of the lens system is slightly offset father from the DMD chip than per design, resulting in a smaller field of view at the focal plane.
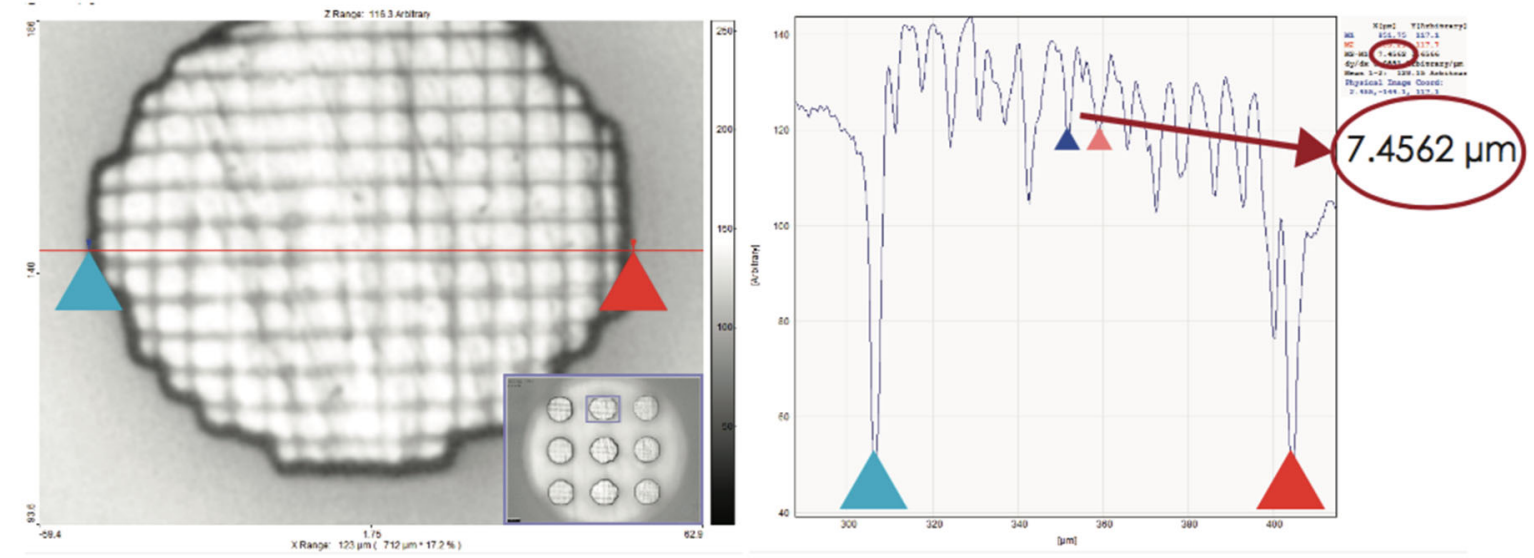

Figure 16 Evaluation of the optical system based upon pixel grid replication on the surface of a test specimen. [13] 


\section{Measurements in additive manufacturing - metal powder consolidation}

With basis in the most common powder based consolidation method, the powder bed fusion manufacturing methods, the following will cover the most prominent measurements as well as highlight the most common the errors that are introduced to the process and which must be understood in order to choose appropriate validation methods during production. For the select errors, methods are proposed to measure them. These errors are:

- Machine Errors

- Deformations due to residual stresses

- Inferior mechanical properties (lack-of-fusion, low densities)

- Surface quality

\section{$\underline{6.1 \text { Machine Errors }}$}

Due to the subsystems that comprise the machine tool for powder bed fusion, some of the errors found in the final products originated from errors contributed to the errors of the equipment itself.

\section{$\underline{6.1 .1 \text { Electro }}$}

The scanner system utilised to move the laser beam over the powder bed consist of two galvanometers. Manoeuvring of these done by an analogue driving current, produced by a digital platform, and the resolution is therefore governed by the resolution achievable by the digital to analogue conversion embedded in the controlling electronics. This circuit is prone to thermal drift, though compensated through a feedback controller, thermal drift will to some extent occur, predominantly from coldstart. Thus, it is important to allow for the machine tool to reach a thermal steady state of the galvanometer system prior to job execution.

\subsubsection{Mechanical}

The process of lowering and recoating the build plate is highly mechanical, and therefore the level of accuracy in displacement of the build plate decides the accuracy of the layers layed out. As the build plate is mounted on a spindle, the error in gradient of the spindle influences the positioning. The spindle error components are reversal error, slack, runout (straightness), angular runout (pitch/yaw/roll), eccentricity, wobble, drift, load capacity (transverse, normal, axial), inertia, jerk and the alignment of the stage to the optical plane of the system. For the re-coater mechanism errors are typically introduced from defects to the re-coater and from the re-coater hitting defects on the workpiece which result in a chatter.

Finally, the galvanometer system is accelerated at extremely high acceleration forces. This may result in overshoot and ringing that is typically seen on worn galvanometer systems and systems operating at excessive speeds.

\subsubsection{Optical}

The laser beam is aligned with the scanner system in a way that allows full use of the scan area, but if any misalignment is present in the system this misalignment influences the positioning and homogeneity of the beam on the powder bed. The scan lens used to focus the beam onto the platform also introduces distortions to both the shape and the scan velocity of the powder bed. Even though most scanner systems are fitted with an $f \theta$-lens, that negates most of the shape distortion and in 
great ensures that the scan velocity is linearly proportional to the angular displacement of the mirrors mounted on the galvanometers, the influence cannot be fully neglected.

\subsection{Deformation due to residual stresses}

One of the most common causes for poor geometrical conformance with specifications of components manufactured by either cladding or powder bed fusion is warp as a result of residual thermal stresses that build up in the workpiece over the duration of the manufacturing of the component. This can to an extent be countered by understanding the consequences of different scan strategies. Choosing a scan strategy to limit that the treated part warps during solidification can alter the outcome from a non-conformance with specifications to conformance. The warpage of the component is caused by a build up of residual stresses as the steep temperature gradient in the normal direction of the workpiece causes the upper layer to expand. As the substrate and solidified material constrains the expansion, the elastic and plastic strain will result in a compressive state. Causing the part to bend towards the heat source, as shown in figure 17 . During cooling the previously plastically compressed upper layer becomes shorter and therefore warping can be observed [14].

\section{Laser Beam}

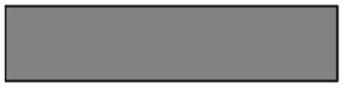

Relaxed State

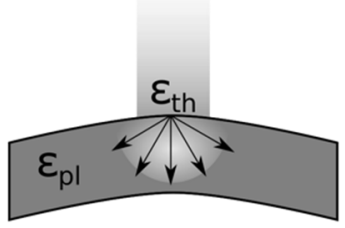

Heating

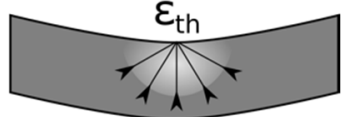

Cooling

Figure 17: Temperature gradient mechanism during heating and cooling

Measurements serve a critical role in validating models used for e.g. optimization of scanning strategies. As presented in the previous section, the localized heating has a great impact on the residual stresses and microstructural formation. The possibility for optimizing the scanning strategy with focus on the final product is being introduced by process modelling groups. Even though the industry has approached this issue, the release is often a stationary pattern that shifts the angle of the hatch lines with a fixed offset. Utilizing numerical modelling and realizing the physical influences on the process allows more specific optimization. As the metal powder consolidation process is a multiphysical process both thermal models, microstructural model, mechanical models and others influences the final product. Therefore an accurately tailored model of the process is cumbersome and CPU intensive [15]. Solving the optimization problem of finding the best scanning strategies, decreasing the overall deformation as a consequence of minimizing the residual stress is naturally even more theoretical. Using a simplified model in order to allow several evaluations in a manageable period of time, can be adopted by subdividing the scan field into smaller squares and applying a pseudo-analytical model together with a simple thermal fitness number, that expresses thermal "inhomogeneity" and degree of consolidation allows this optimization of scan strategy in a short time frame [16]. 


\section{Т}

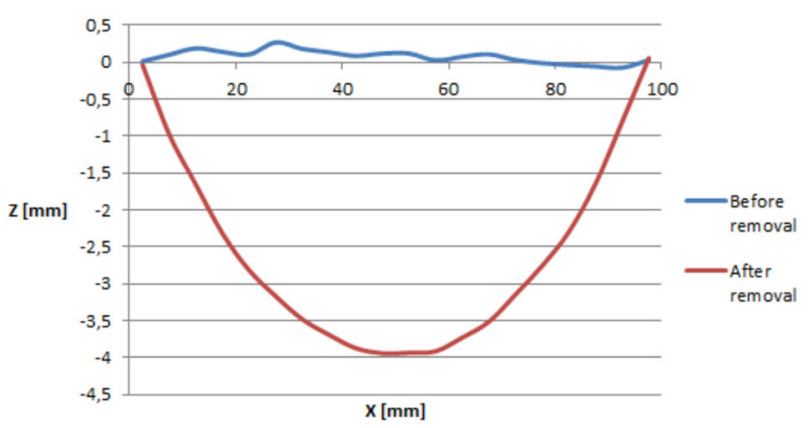

Figure 18: Beam with traditional parallel scan strategies, maximal deformations of $4 \mathrm{~mm}$ is measured from form measurement of the top-surface. [16]

In Fig 18 a beam was consolidated using traditional scan strategies, and the deformation after being removed from the build plate was measured with a coordinate measurement machine. Here the deformation due to the build up of residual stresses is visible. Using an optimized scan strategy based on the thermal fitness number, and therefore a simplified model lead to the model seen in fig 19 . Here the deformation of the beam has been reduced by a factor of 10 , validated by form measurements of the samples, figures 18-19.

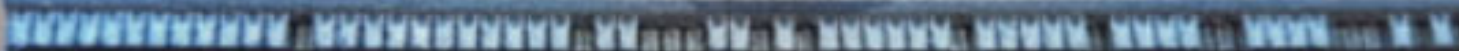

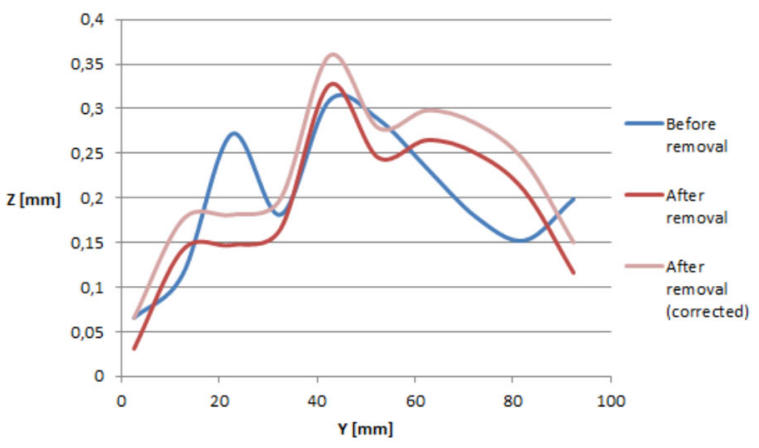

Figure 19. Beam produced with an optimized scan strategy, maximal deformation of $0.35 \mathrm{~mm}$ from form measurement of the top-surface [16]

\subsection{Density (inclusions, porosities, voids)}

When metal additive manufacturing is employed for industrial grade component manufacturing, ensuring that the method is capable of producing components with mechanical properties similar to those from traditional manufacturing is of essence. The quality of the produced parts is thus in great defined from the density of the part. Several proprietary powder bed fusion systems claims a density of close to $100 \%$. Verification of the density is key to validating if the component has the expected 
performance characteristics, and will often have to be carried out as part of the quality assurance, here three methods will be described

$\begin{array}{ll}- & \text { Archimedes-method } \\ \text { - } & \text { Computed Tomography (CT) } \\ \text { Optical Measurements Technologies }\end{array}$

\subsubsection{Archimedes method}

The method of Archimedes is one of the more straightforward methods of verifying the density of a part. The mean density of the entire part is obtainable regardless of the complexity of the part. The method requires a highly accuracy scale, and a liquid with a known density. The part is consequently measured in air, $m_{a}$, and the again when lowered into the liquid, $m_{l}$. The part density is the defined from equation 1 , and can be compared to the nominal density of the bulk material.

$$
\rho_{p}=\frac{m_{a}}{m_{a}-m_{l}} \cdot \rho_{l}
$$

\subsubsection{Computed Tomography (CT)}

Despite the robust and straightforward way of the Archimedes method in determining the average density of the part, it does not yield any information about the location or size of the individual porosities yielding a not fully dense part. To obtain this kind of information other methods have to be applied.

$\mathrm{X}$-ray CT scanning is a suitable non-destructive way of creating a 3D image of the internal structure of the component and its contained porosities. This method relies on the emission of $x$-rays. These are very high frequency and short length waves, that will penetrate solids, and much like the shadow cast by a hand by visible light, the geometry of the intercepting component can be recorded with a detector. The detector records the intensity of the x-rays after they penetrated the part, and is thereby capable of locating voids or other inclusions obstructing the passage of the $x$-rays. Each image captured by this method is by nature $2 \mathrm{D}$, and will therefore give a $2 \mathrm{D}$ position of the inhomogeneity of the metal part. From the 2D image stack, the true 3D location and size of voids and inclusions can be mapped by correlating the image stack. Correlating the images allows a $3 \mathrm{D}$ reconstruction of the part, as shown in figure 20, describing the entirety of the CT scanned object. For this method the spatial resolution is inversely coupled to the size of the volume investigated. Therefore it can be hard to obtain information of very small defects. Studying the 3D model, information about the location, size and shape of the voids is achievable as shown in figure 21 , where a sample has been scanned to reveal internal porosities.

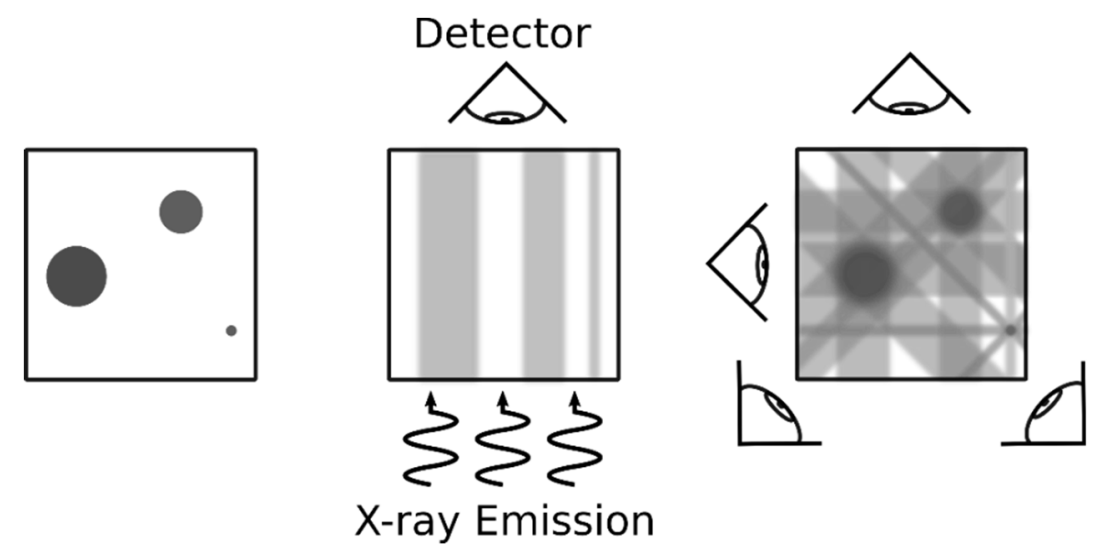




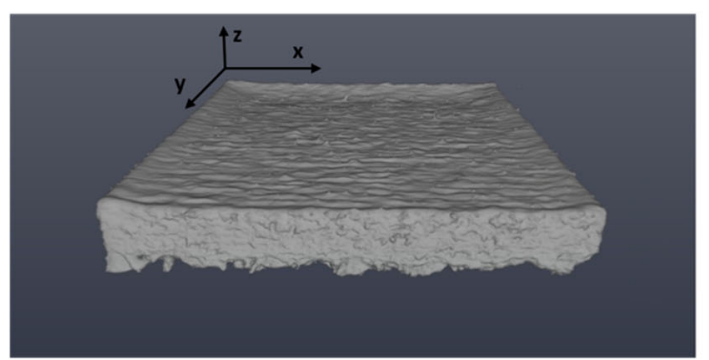

a) CT-reconstruction

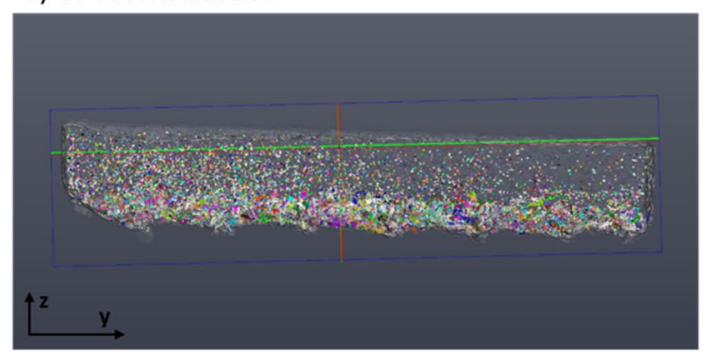

b) Identified pores visualized in Avizo

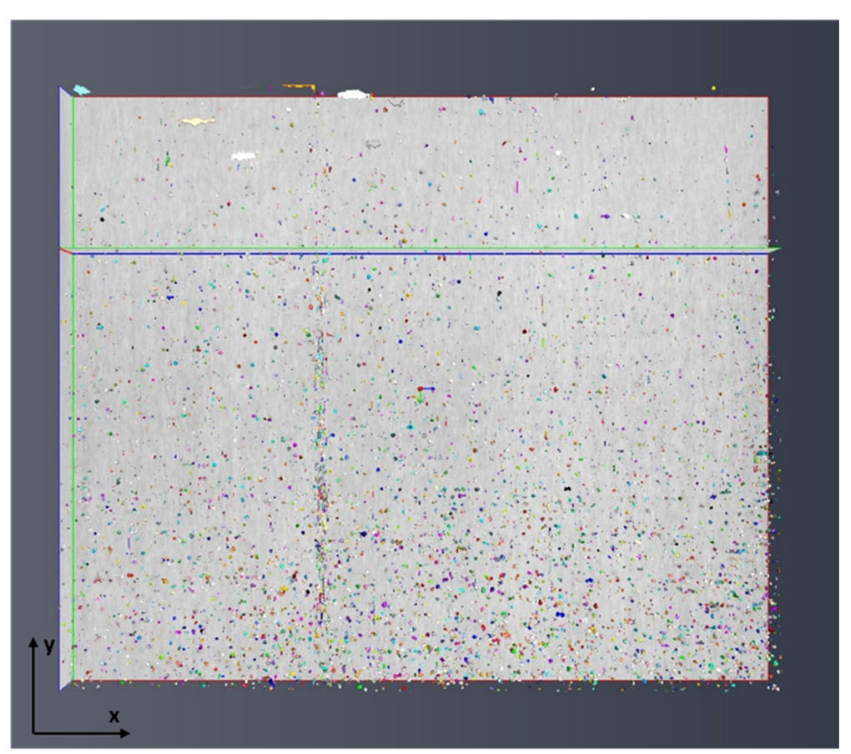

c) Identified pores visualized in Avizo in the reduced area

Figure 21: Reconstruction of a CT scan of a $10 \times 10 \mathrm{~mm}$ diameter, $1.5 \mathrm{~mm}$ tall flat sample showing internal porosities [17]

\subsubsection{Optical Microscopy}

Optical Microscopy is a destructive method for obtaining information about porosities of the component. Through this method, the information obtained relies on the diffusive reflectance of the surface, and the specimen must therefore become optically smooth. Smooth surfaces are achieved by making a cross sectional cut, and polishing until the surface is adequate for optical microscopy. Cutting the part into slices of a chosen thickness, puts this method in the destructive category.

Investigating the polished surface allows for detection of voids or inclusions, see figure 22. OM does not have the limiting spatial factor known from CT scans, as spatial investigation can be carried out by serial-sectioning. The $2 \mathrm{D}$ images captured allows detection of smaller defects than the $\mathrm{CT}$, but due to the polishing and cutting more time consuming. 


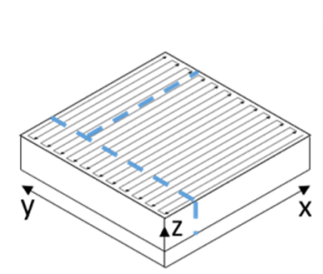

a) Sample cut

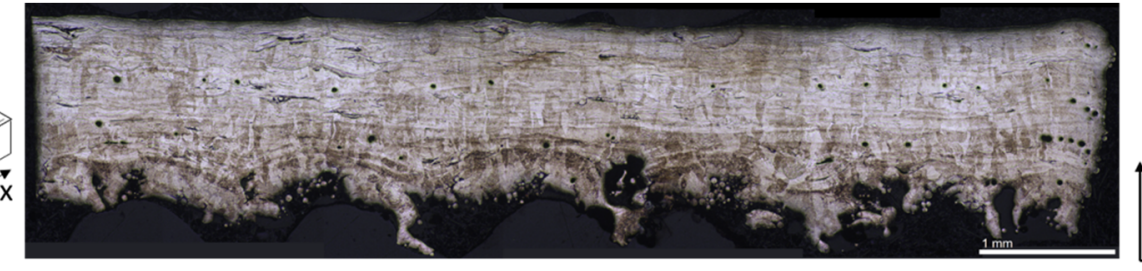

b) $x-z$ cross section
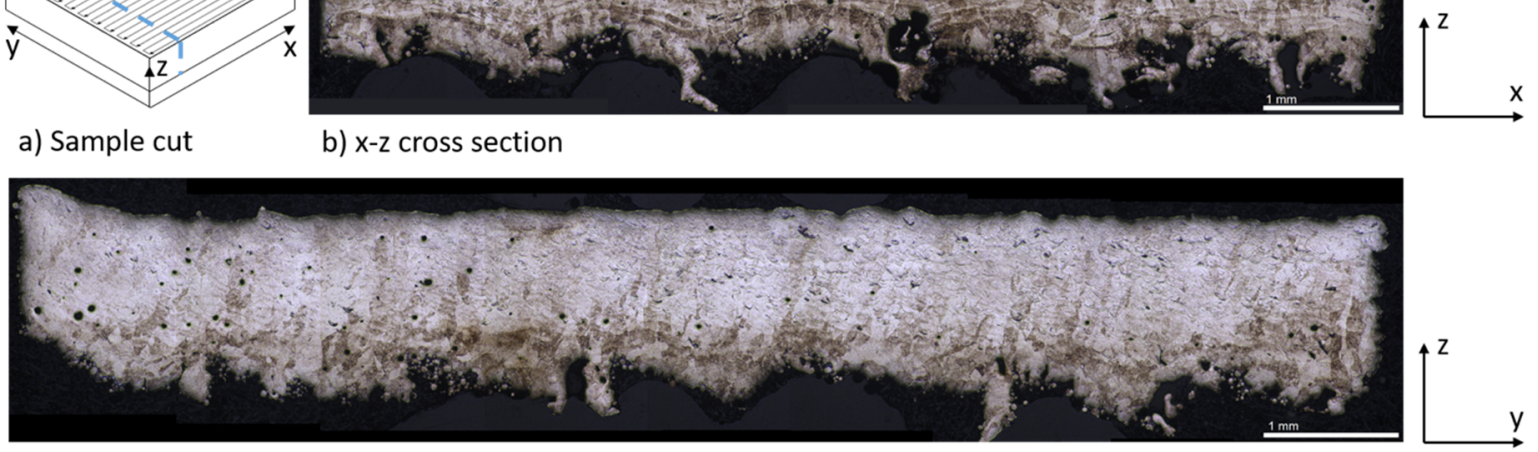

c) $y-z$ cross section

Figure 22: (A) High resolution optical microscopy montage of a 10x10 mm diameter, $1.5 \mathrm{~mm}$ tall flat sample showing the morphology of two populations of voids (a) Sectioning, (b) $x-z$ section from CT reconstruction, (c) $y$-z cross section. [17]

Aside from observing voids in the cross section, optical microscopy is also very capable of revealing geometry surface textures and optical material properties revealed after etching.

Using an etching agent on the polished surface will reveal the microstructure present in that exact cross section and looking at the AM part both along the scan direction and across will reveal a difference in microstructure according to material, scan velocity, power and scan strategy.

\subsection{Surface quality}

The surface quality of the consolidated powders is very complex as a consequence of the selectively melting and resolidification, and therefore has very poor surface characteristics compared to other processes as the surface is elongated melt pools. These are challenging to measure due the inhomogeneity and residue left by spatter.

\subsubsection{Topology inspection of surfaces}

Achieving sound measurements of the surface topology is not yet fully achievable, and most like the best fit is found in a combination of different methods.

Confocal Microscopy (CM), Focus Variation (FV) microscopy, Coherence Scanning Interferometry (CSI) and XCT are among the methods that can be used to obtain knowledge about the topology of the powder bed fusion parts. As the surface is prone to having irregular optical features the optical measurements provides differences related to the approach used. The discrepancy between the mentioned measurements methods for the same topography are presented in table 2 , and a qualitative overview is given in fig 23 .

It is revealed from table 2, that the discrepancy amongst the different methods is high, and must therefore be applied only with thorough understanding of the specimen and the measurement method used. 
Table 2: Profile discrepancy between instrument pairs. Percentage of the profile length where the confidence interval does not intersect. [18]

\begin{tabular}{lllllll}
\hline & $\mathrm{CM} / \mathrm{CSI}$ & $\mathrm{CM} / \mathrm{FV}$ & $\mathrm{CM} / \mathrm{XCT}$ & $\mathrm{CSI} / \mathrm{FV}$ & $\mathrm{CSI} / \mathrm{XCT}$ & $\mathrm{FV} / \mathrm{XCT}$ \\
\hline Discrepancy \% & 51.7 & 61.9 & 48.5 & 57.4 & 42.9 & 48.8 \\
\hline
\end{tabular}

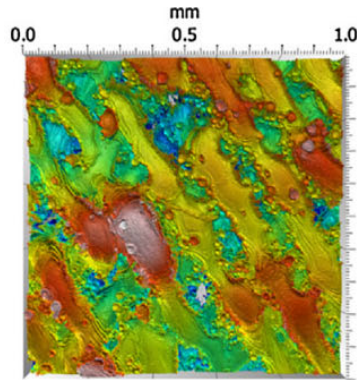

a)

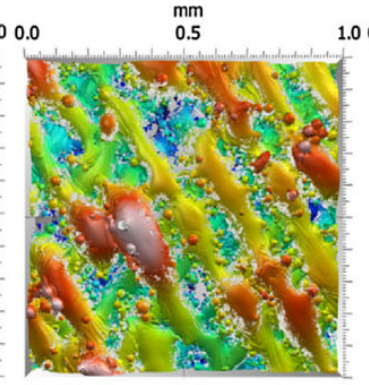

b)

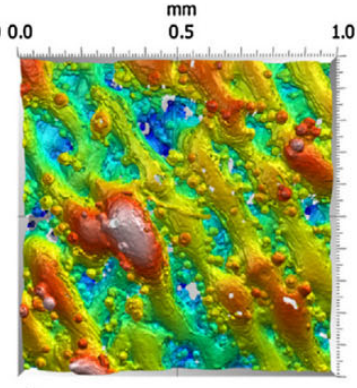

c)

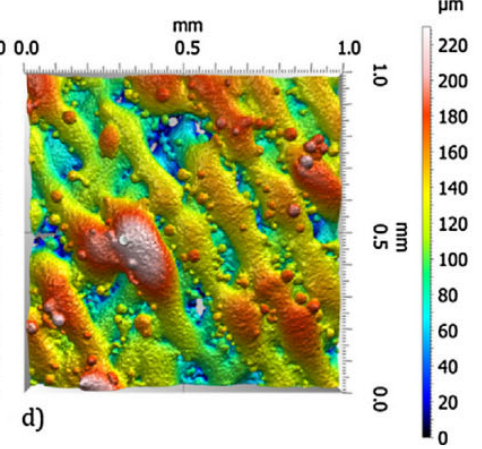

Figure 23, Reconstructed portions of aligned topographies (top views, height-based colouring); (a) CM; (b) CSI; (c) FV; (d) XCT [18]

\section{Summary}

In principle, measurements in additive manufacturing are only measurements. In this chapter we have tried to introduce a model for linking the digital and physical process chain related to additive manufacturing. These two process chains both introduce errors and variations into the final component manufactured. The chapter describes in detail two industrially adapted additive manufacturing processes: photo polymerization and metal powder consolidation. For each of these process families, measurements and their challenges related to machine errors, material properties, shape deformations and surface quality are described and discussed. 
[1] "Photopolymerization and Photo-Cross-Linking," in Photoinitiators for Polymer Synthesis, Weinheim, Germany: Wiley-VCH Verlag GmbH \& Co. KGaA, December 2012, pages 3-9.

[2] C. Decker, "Photoinitiated crosslinking polymerisation," Progress in Polymer Science, volume 21, number 4, pages 593-650, January 1996, issn: 00796700.

[3] ISO 8062; ISO/TR 14638 Geometrical product specifications (GPS) - Dimensional and geometrical tolerances for moulded parts - Part 3: General dimensional and geometrical tolerances and machining allowances for castings

[4] DS/CEN ISO. Geometrical product specifications (GPS) - Masterplan (DS/CEN/CR ISO 14638) 1997.

[5] S. Kalpakjian, S. R. Schmid, (2010), Manufacturing Engineering and Technology, Pearson, Ch. 17. ISBN: 9810681445

[6] Gibson, D. W. Rosen, B. Stucker, (2010), Additive Manufacturing Technologies: 3D Printing, Rapid Prototyping, and Direct Digital Manufacturing, Springer, section 5.6.2. ISBN: 1493921134

[7] Gibson, D. W. Rosen, B. Stucker, (2010), Additive Manufacturing Technologies: 3D Printing, Rapid Prototyping, and Direct Digital Manufacturing, Springer, section 5.3.1. ISBN: 1493921134

[8] Ahmad, A. Handbook of Optomechanical Engineering, Second Edition, 2017, CRC Press, ISBN 9781498761482

[9] I. Gibson, D. W. Rosen, and B. Stucker, "Photopolymerization Processes," in Additive Manufacturing Technologies, Boston, MA: Springer US, 2010, pages 78-119.

[10] Eschl, J., Blumenstock, T., Eyerer, P. Comparison of the Curing Process of Epoxy and Acrylate Resins for Stereolithography by Means of Experimental Investigations and FEM-Simulation, 1999 SOLID FREEFORM FABRICATION PROCEEDINGS; 453-460; Solid freeform fabrication symposium.

[11] Moothanchery, M. et al. Real-time shrinkage studies in photopolymer Ims using holographic interferometry. 2012, Proc. SPIE 8437, 84370I. Brussels Belgium. 16-19 April.

[12] Technical Paper: Stress Crazing On Acrylic Surfaces And Its Causes, July 2007, Aristech Acrylics LLC

[13] Ribó, M.M. 3D Printing of Bio-inspired Surfaces, M.Sc Dissertation, Technical University of Denmark, dept. of mechanical engineering October 2017

[14] Kruth, J. P., et al. Selective laser melting of iron-based powder. Journal of Materials Processing Technology 2004, 149(1-3), 616-622.

[15] Saad A. et al. Laser powder-bed fusion additive manufacturing: Physics of complex melt flow and formation mechanisms of pores, spatter, and denudation zones, In Acta Materialia, Volume 108, 2016, Pages 36-45, ISSN 1359-6454 
[16] Mohanty, S. et al. Achieving dimensional tolerances in metal additive manufacturing via numerical model based process optimization, Dimensional Accuracy and Surface Finish in Additive Manufacturing, KU Leuven, BE, October 2017

[17] Valente, E. H. Heat and surface treatment of 3D printed titanium, M.Sc. dissertation, Technical University of Denmark, dept. of mechanical engineering June 2017

[18] Thompson, A., Senin, N., Giusca, C., \& Leach, R. (2017). Topography of selectively laser melted surfaces: A comparison of different measurement methods. CIRP Annals, Manufacturing Technology, 66(1), 543-546 